\title{
Detachment of hard ticks (Acari: Ixodidae) from hunted sika deer (Cervus nippon)
}

\section{Takashi Tsunoda}

Laboratory of Applied Entomology and Zoology, Faculty of Horticulture, Chiba University

648 Matsudo, 271-8510 Japan

Present address: Department of Vector Ecology and Environment, Institute of Tropical Medicine, Nagasaki University, 1-12-4 Sakamoto, Nagasaki 852-8523, Japan

E-mail: tsunoda@nagasaki-u.ac.jp

Telephone number: +81958197809

Fax number: +81958197812 
Ixodid ticks attach to their host to suck blood meal, with attachment often lasting 1-2 weeks (Balashov 1972). However, when a host dies, ticks gradually detach and leave. The distribution of tick species and genders on a host may change as a function of time after the host's death. Since hundreds or thousands of ticks often detach from a dead host, it is difficult to count the number of ticks that detach from a host after its death. Little is known about detachment of ixodid ticks from dead hosts.

The hypostome of an attached ixodid tick penetratees its host for a blood meal (Arthur 1962; Moorhouse 1967). A cement-like substance from the salivary glands glues the mouthparts firmly into the host to ensure continuous attachment during the long feeding times (Kemp et al. 1982). It is assumed that the fraction of ixodid ticks on a host with their mouthparts glued to the host, relative to the fraction that have detached and left, gradually decreases after the death of the host.

Sixteen species of ixodid ticks have been reported to be ectoparasites of sika deer in Japan (Ohdachi et al. 2009). In the Boso Peninsula of Japan, the bush tick Haemaphysalis longicornis is the dominant parasite of sika deer in the summer and Haemaphysalis megaspinosa is dominant in the winter (Mori et al. 1995), with adults of both species abundant in March (Tsunoda 2007).

Ticks were obtained from 13 sika deer, Cervus n. nippon, shot in Amatsu, the eastern part of Kamogawa City in the Boso Peninsula in central Japan, from late February to early March 1999. Hunters put each deer in a large polyvinyl plastic bag immediately after they shot it. Cadavers were kept in the bags until the hunters dissected them 1 to $3 \mathrm{hr}$ after culling. Fur from the head, legs, and body were put in another polyvinyl plastic bag, brought to a laboratory $3 \mathrm{hr}$ later, and kept in a $-20^{\circ} \mathrm{C}$ freezer to kill ticks.

Fur was kept at $25^{\circ} \mathrm{C}$ for at least for $12 \mathrm{hr}$ before collection of ticks. For collection from a deer body surface, ticks were collected from either the left or right side of the head and body and from the legs. Ticks were also collected from the polyvinyl plastic bag. A tick with its hypostome inserted into the host skin (designated attached) was distinguished from a tick that was detached and on the body surface. In addition, female ticks were classified in three groups based on their blood feeding stage: engorged (i.e. swollen into a globular shape and with a black color), partially-engorged (i.e. swollen, but not into a globular shape, and with a brown color), and unfed (i.e. unfed or little feeding, although these were difficult to discriminate).

A total of 3,954 adult, 4,495 nymph and 83 larva Haemaphysalis ticks were collected from 13 sika deer. The most abundant adult ticks collected were $H$. megaspinosa, and smaller numbers of $H$. longicornis, $H$. flava, $H$. kitaokai, and $H$. 
cornigera (Table 1). H. ias is a synonym of H. cornigera (Takada 1990) However, 92 Haemaphysalis females, most of them were partially-engorged, could not be identified because they had lost their capitulum when they were pulled out of their hosts with forceps. In addition, 35 Ixodes ovatus adults and 1 Amblyomma testudinarium nymph were also collected.

Males were more abundant than females for the H. longicornis, $H$. megaspinosa, and $H$. flava ticks collected (Fig. 1A-C) but not for H.kitaokai (Fig. 1D) (Wilcoxson signed-rank test, $H$. longicornis: $\mathrm{Z}=91, P<0.01 ; H$. megaspinosa: $\mathrm{Z}=91, P<0.001 ; H$. flava: $\mathrm{Z}=90, P<0.01$; H.kitaokai: $\mathrm{Z}=62.5, P=0.07)$. There was a significant difference in the sex ratio of $H$. megaspinosa, $H$. longicornis, and $H$. kitaokai ticks that were attached or detached (Table 2a). A greater fraction of the male than female $H$. megaspinosa, H. longicornis, and H. kitaokai were detached (Fig. 2).

A generalized linear model (GLM) with a binomial distribution and a log link function was used to test whether detached or attached ticks were significantly different in terms of sex ratio and blood feeding stage. For feeding stage analysis, engorged ticks were grouped with partially-engorged ticks because the number of engorged ticks was small. Overdispersion was resolved by use of a quasi-binomial distribution. Model comparisons were analyzed using the F-test. There was a significant relationship between whether $H$. megaspinosa and $H$. longicornis ticks were attached or detached and their feeding stage (Table 2b). More detached $H$. longicornis and $H$. megaspinosa unfed female ticks were collected than engorged and partially-engorged females (Fig. 3). However, for H. kitaokai, there was no significant relationship between whether female ticks were attached or detached and their feeding stage.

More $H$. megaspinosa, $H$. longicornis, $H$. flava, and $H$. kitaokai male than female ticks were collected on the sika deer in this study. It has been reported that there were more $H$. megaspinosa and $H$. flava male than female ticks on wild boar, and more $H$. longicornis male than female ticks on Reeve's muntjac (Tsunoda 2012). Metastriata ticks mate on their host and prostriata ticks mate both on and off their host (Sonenshine 1991). Although some prostriata male ticks can inseminate up to 3 females (Graf 1978; Yunker et al. 1991), male ticks of the metastriata species Amblyomma americanum can inseminate up to 37 females (Gladney and Drummond 1970). The sex ratio of metastriata ticks, with more males than females on hosts, suggests that the male's reproductive strategy may be to remain longer on their hosts to enable them to encounter and mate with more females.

Although more $H$. longicornis and $H$. kitaokai females than males were attached, more $H$. megaspinosa, $H$. longicornis, $H$. flava, and $H$. kitaokai males than females 
detached after their host died. These results indicated that $H$. megaspinosa, $H$. longicornis, $H$. flava, and $H$. kitaokai male ticks detached sooner than female ticks after their host died.

Ixodes dammin (the same species as I.scapularis) eggs are fertilized by sperm from males a second copulation when a female copulates with two males (Yuval and Spielman 1990). Although there is no information on sperm displacement in metastriata, it should be advantageous for males to guard females after insemination until females detach from the host. In general, female ticks that have larger blood meals lay more eggs (Sonenshine 1991). In Rhipicephalus appendiculatus, a male helps its female mate feed by modulating the host immune response during co-feeding (Wang et al. 1998). However, if a host is dead, the male does not have to stay attached long because it is difficult for females to take a blood meal. Therefore, it may be beneficial for males to detach from a dead host and wait for a new host.

Acknowledgements I thank the Chiba Hunting Federation, particularly gamekeepers and hunters, Nature Conservation Division of Environmental and Community Affairs Department in Chiba Prefecture, K. Ochiai, and Y. Kaneshiro for their collaboration in the collection of samples. I appreciate M. Kawashima, D. Hirano, T. Shintaku, M. Oizumi, R. Fukuda, T. Ito, T. Torii, H. Takahashi, N. Haruyama, and M. Miyamoto for their help in collecting ticks from deer. 
Figure legends

Figure 1. Number of male and female ticks per deer. (A) Haemaphysalis longicornis, (B) H. megaspinosa, (C) H. flava, (D) H. kitaokai. The mean \pm SE of each population is shown. Asterisks show significant difference: ${ }^{*} p<0.01$, $* * * p<0.001$; NS indicates lack of significance at $p>0.05$.

Figure 2. Percent of male and female ticks that were detached or attached. (A) Haemaphysalis longicornis, (B) H. megaspinosa, (C) H. flava, (D) H. kitaokai. The numbers in parenthesis denote the total numbers of ticks.

Figure 3. Percent of fed (engorged + partially-engorged) and unfed ticks that were detached or attached. (A) Haemaphysalis longicornis, (B) H. megaspinosa, (C) $\mathrm{H}$. kitaokai. The numbers in parenthesis denote the total numbers of ticks. 
Table 1. Adult Haemaphysalis and Ixodes ticks collected from 13 sika deer in the Boso Peninsula

\begin{tabular}{lrrrr}
\hline \multirow{2}{*}{ Species } & Male & \multicolumn{3}{c}{ Female } \\
\cline { 2 - 5 } & & Engorged & Partially-engorged & Unfed \\
\hline H.longicornis & 244 & 1 & 63 & 70 \\
H.megaspinosa & 1982 & 30 & 649 & 388 \\
H.flava & 91 & 0 & 6 & 13 \\
H.kitaokai & 176 & 9 & 48 & 53 \\
H.cornigera & 1 & 0 & 1 & 0 \\
H.sp. & 0 & 4 & 84 & 4 \\
I.ovatus & 16 & 5 & 11 & 3 \\
I.sp. & 0 & 0 & 2 & 0 \\
\hline
\end{tabular}


Table 2. Results of GLM for (A) sex ratio of four species of ticks and (B) blood feeding level of three species

\begin{tabular}{llclll} 
(A) & \multicolumn{1}{l}{} \\
\hline Response variable & Explanatory variable & Estimate & SE & \multicolumn{1}{l}{ T } & P \\
\hline H. megaspinosa & Intercept & 0.69 & 0.06 & 11.61 & $<0.001$ \\
& Condition* & -0.76 & 0.20 & -3.85 & $<0.001$ \\
H.longicornis & Intercept & 0.78 & 0.13 & 5.85 & $<0.001$ \\
& Condition* & -1.63 & 0.42 & -3.92 & $<0.001$ \\
H. flava & Intercept & 1.49 & 0.25 & 5.88 & $<0.001$ \\
& Condition* & 0.91 & 1.05 & 0.87 & 0.40 \\
H. kitaokai & Intercept & 1.07 & 0.22 & 4.83 & $<0.001$ \\
& Condition* & -2.15 & 0.43 & -4.97 & $<0.001$ \\
\hline
\end{tabular}

*Condition: detached or attached.

(B)

\begin{tabular}{llclcl}
\hline Response variable & Explanatory variable & Estimate & SE & \multicolumn{1}{l}{ T } & P \\
\hline H. megaspinosa & Intercept & 1.63 & 0.38 & 4.28 & $<0.001$ \\
& Condition* & -1.33 & 0.40 & -3.32 & $<0.005$ \\
H.longicornis & Intercept & 1.05 & 0.58 & 1.82 & 0.09 \\
& Condition* & -1.57 & 0.65 & -2.41 & 0.03 \\
H. kitaokai & Intercept & 0.14 & 0.35 & 0.41 & 0.69 \\
& Condition* & 0.25 & 0.48 & 0.53 & 0.61 \\
\hline
\end{tabular}

*Condition: detached or attached. 


\section{References}

Arthur, D (1962) Ticks and Disease. Row, Peterson and Company, Evanston, Illinois.

Balashov, YS (1972) Bloodsucking ticks (Ixodoidea) - vectors of disease of man and annimals (English translation). Misc. Publ. Entomol. Soc. Amer. 8: 163-376.

Gladney, WJ, Drummond, RO (1970) Migration of male lone star ticks on the host in relation to mating. J Econ Entomol 63: 1214-1216.

Graf, JF (1978) Copulation, nutrition et ponte chez Ixodes ricinus L. (Ixodoidea: Ixodidae) - 2e partie. Bull Soc Entomol Suisse 51: 241-253.

Kemp, D, Stone, B, Binnington, K (1982) Tick attachment and feeding: Role of the mouthparts, feeding apparatus, salivary gland secretions and the host response. In: Obenchain, F,Galun, R (ed), Physiology of Ticks. Pergamon Press, Oxford pp 119-168.

Moorhouse, D (1967) The attachment of some ixodid ticks to their natural hosts. Proc. 2nd Int. Congr. Acarol.

Mori, K, Tsunoda, T, Fujimagari, M (1995) Ixodid ticks on Sika deer Cervus nippon Temminck in Chiba Prefecture. . Jpn J Sanit Zool 46: 313-316 (in Japanese with English abstract).

Ohdachi, DS, Ishibashi, Y, Iwasa, M, Saitoh, T (2009) The Wild Mammals of Japan. SHOUKADOH Book Sellers, Kyoto.

Sonenshine, DE (1991) Biology of ticks. Vol. 1. Oxford University Press, New York.

Takada, N (1990) Byogendaniruizufu. Kinpodo, Kyoto.

Tsunoda, T (2007) Interspecific and intraspecific associations of two species of hard ticks, Haemaphysalis longicornis and Haemaphysalis megaspinosa, in relation to questing site. J Parasitol 93: 531-541.

Tsunoda, T (2012) Tick fauna from middle- and large-mammals in Chiba Prefecture, central Japan. J Nat His Mus Inst Chiba 12: 33-42 (in Japanese with English summary).

Wang, H, Paesen, GC, Nuttall, PA, Barbour, AG (1998) Male ticks help their mates to feed. Nature 391: 753-754.

Yunker, CE, Andrew, HR, Norval, RAI, Keirans, JE (1991) Interspecific attraction to male-produced pheromones of two species of Amblyomma ticks (Acari: Ixodidae). J Insect Behav 3: 557-567.

Yuval, B, Spielman, A (1990) Sperm precedence in the deer tick Ixodes dammini. Physiol Entomol 15: 123-128. 
Figure 1.

A)

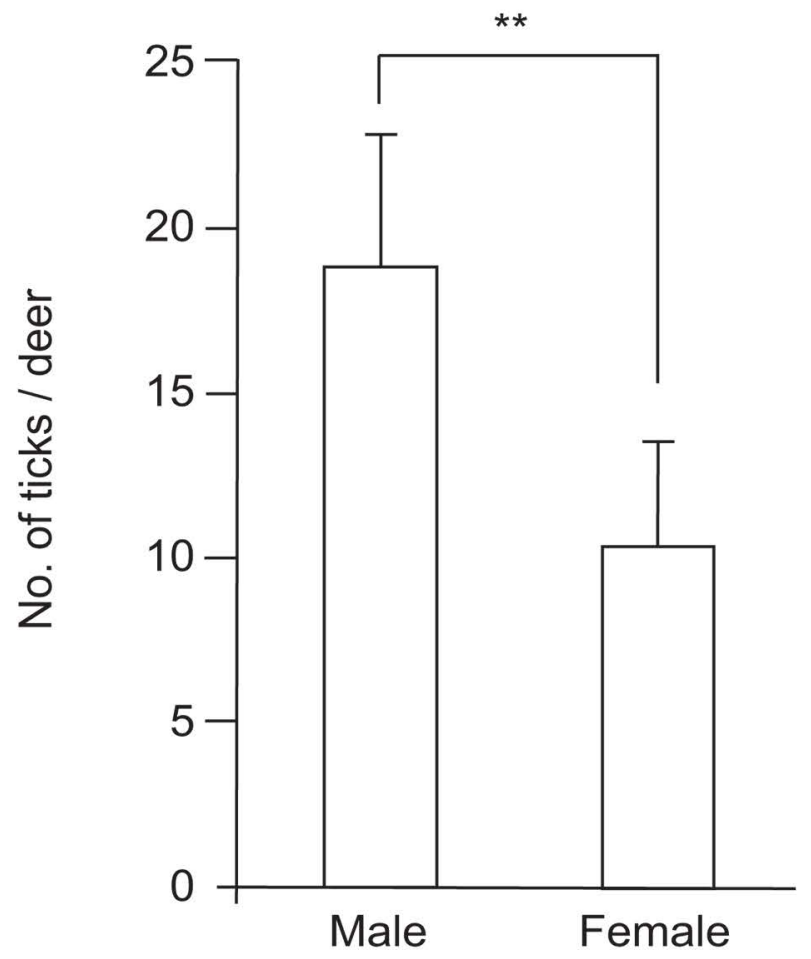

C)

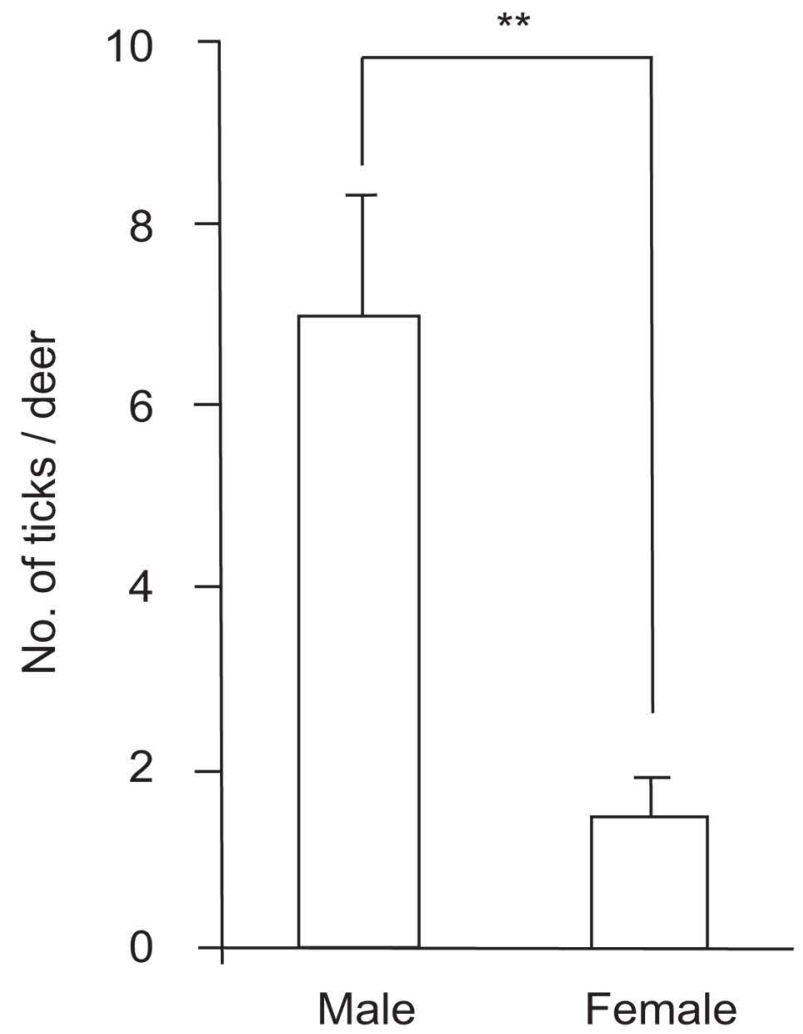

B)

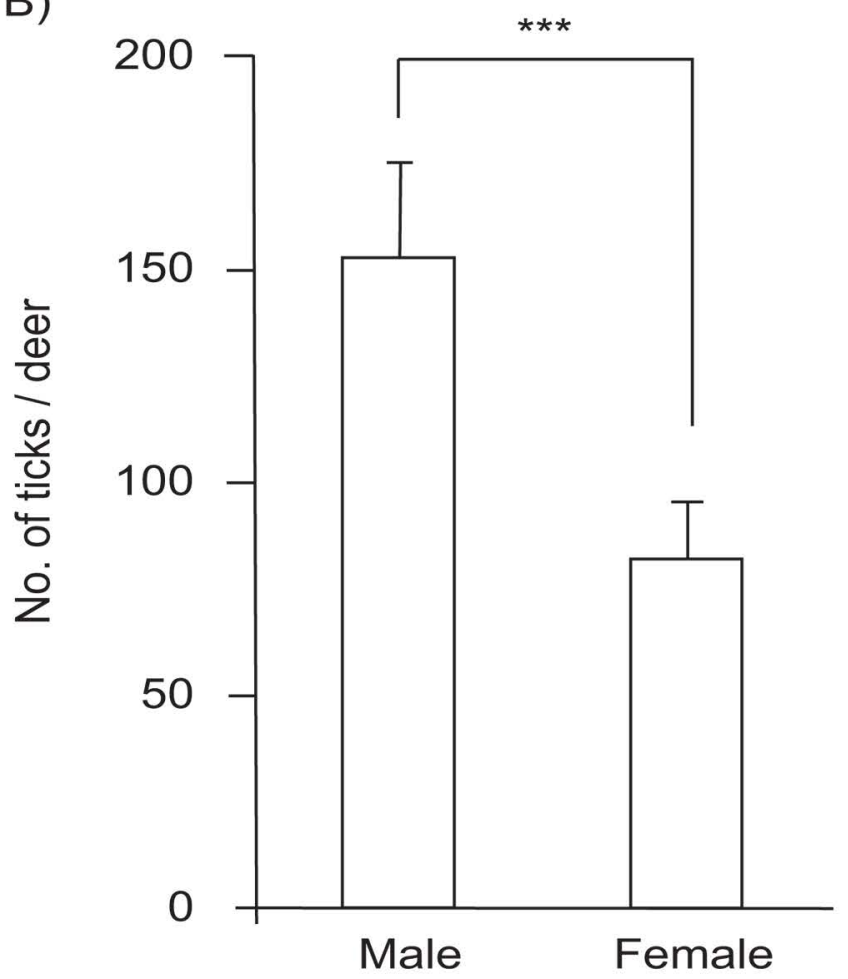

D)

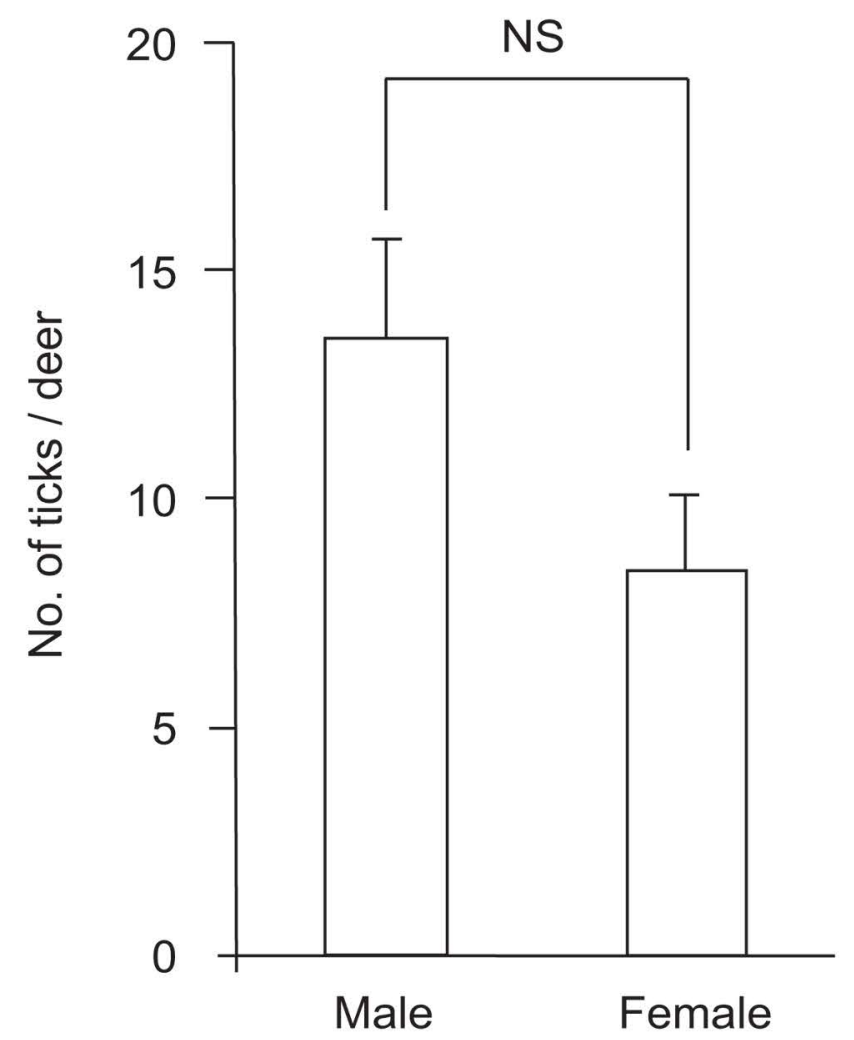


Figure 2.

A)

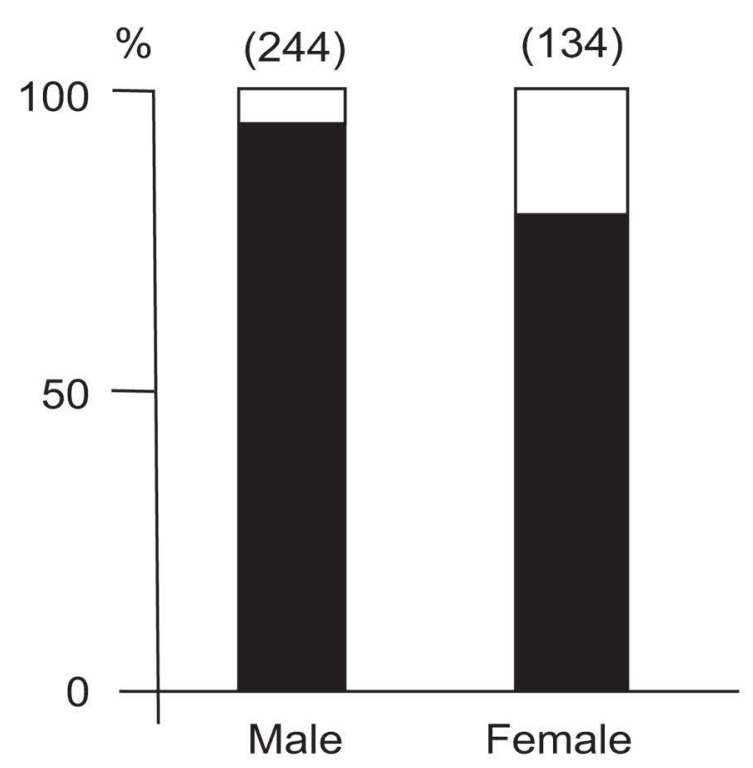

C)

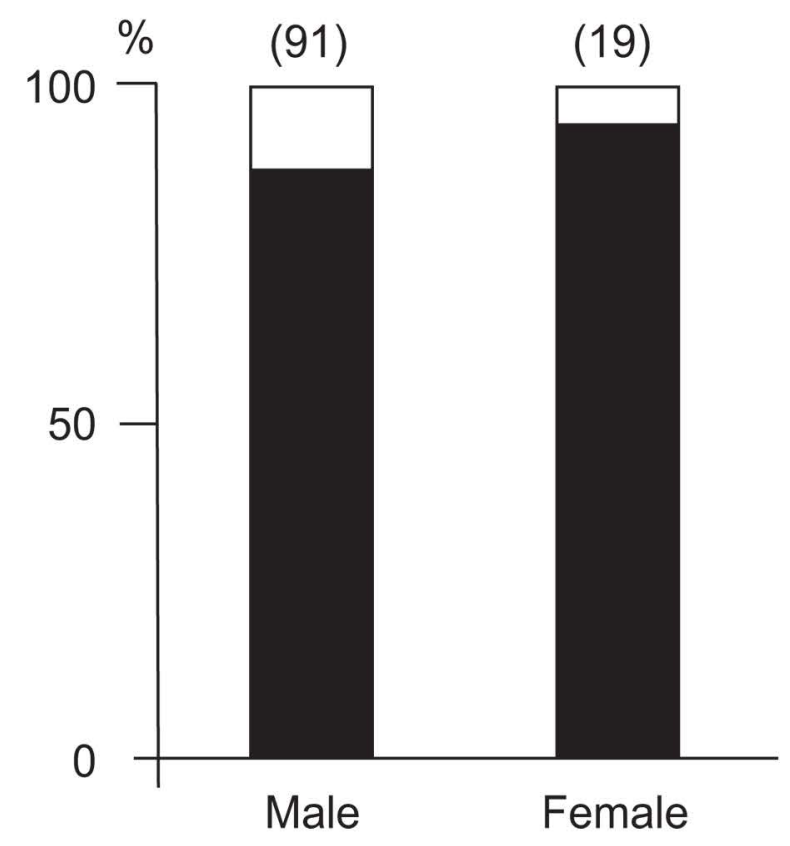

B)

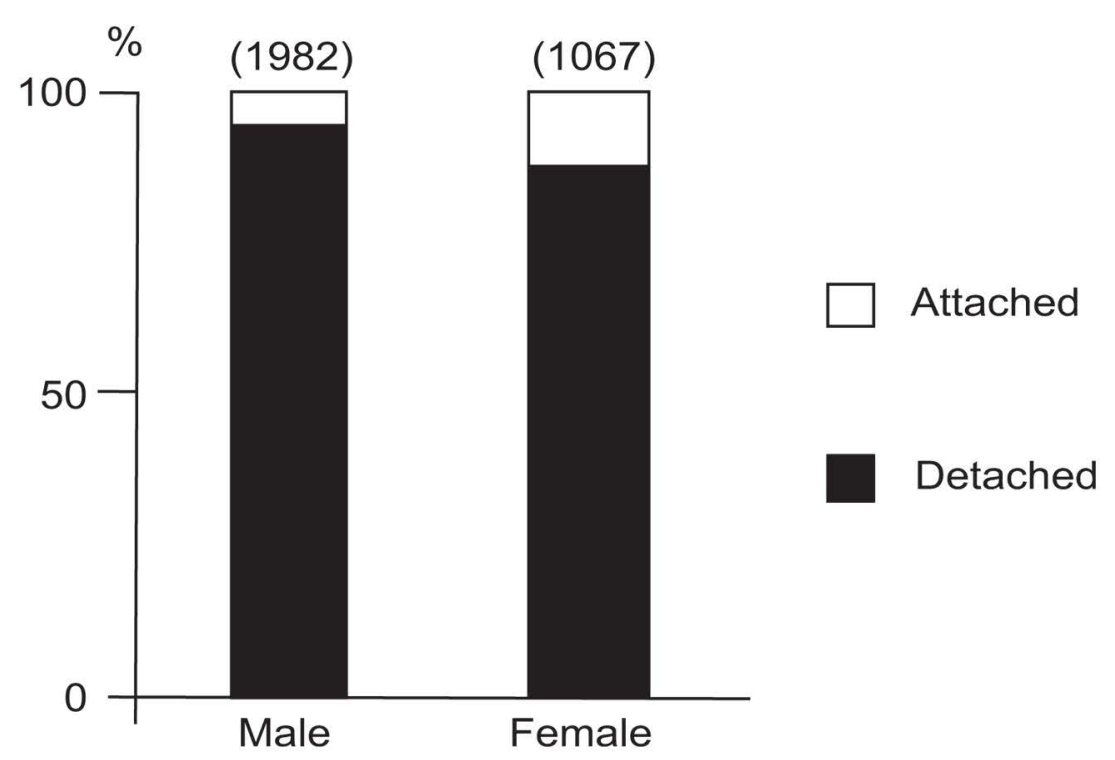

Attached

D)

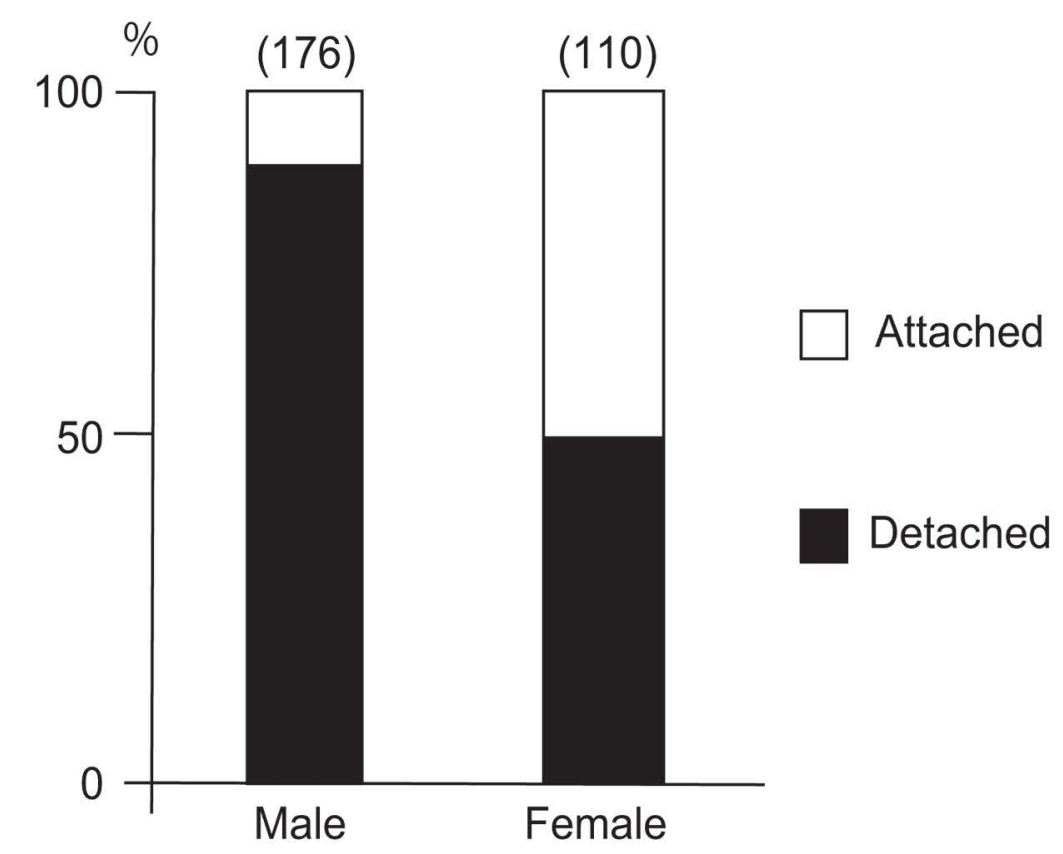


Figure 3.

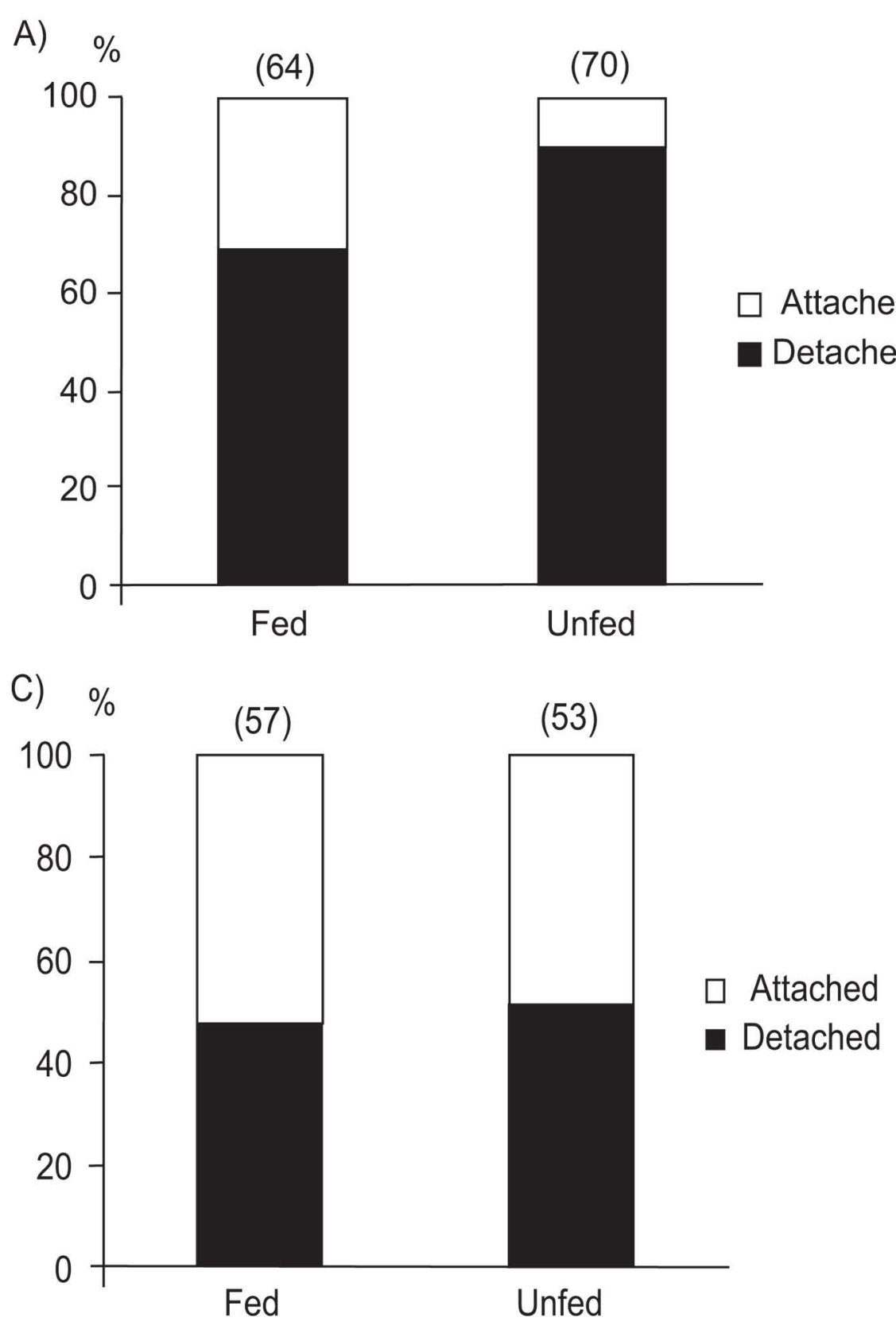

B)

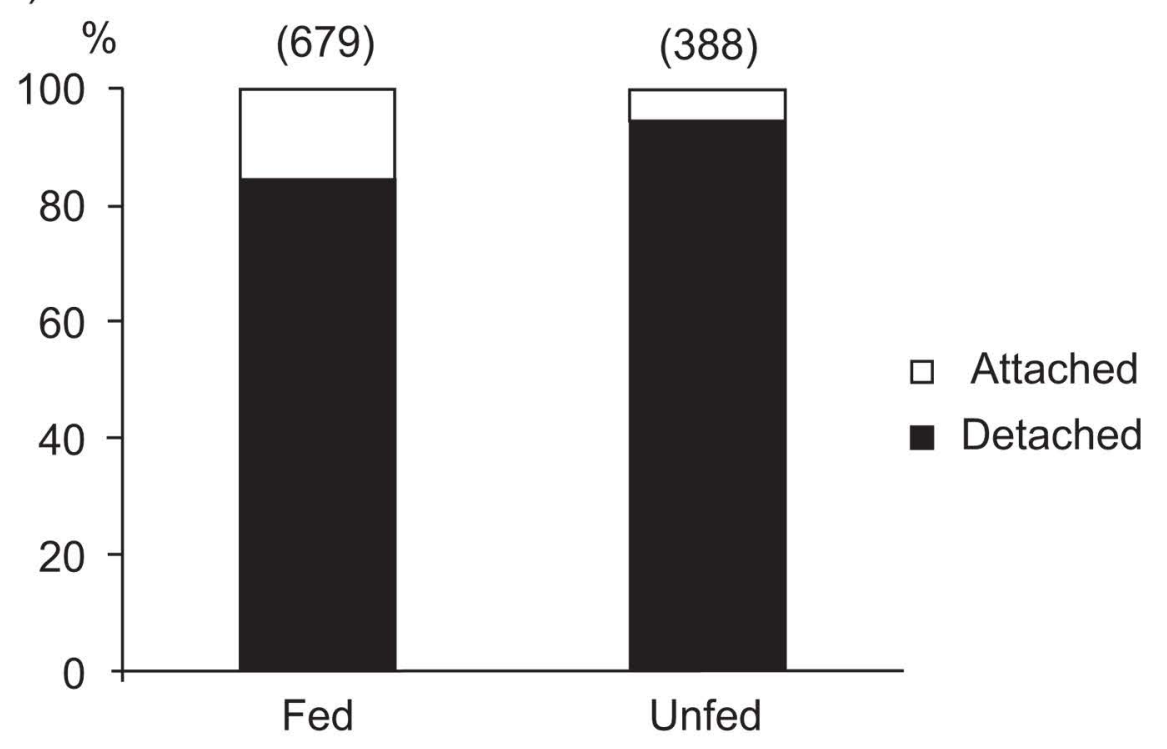

